Collection SFN 8 (2007) 1-9

(C) EDP Sciences, Les Ulis

DOI: $10.1051 / \mathrm{sfn}: 2007002$

\title{
Introduction à la diffusion de neutrons
}

\section{J. Teixeira}

Laboratoire Léon Brillouin (CEA/CNRS), CEA Saclay, 91191 Gif-sur-Yvette Cedex, France

L'étude de la matière condensée au niveau atomique et moléculaire s'appuie souvent sur les résultats de l'interaction avec des particules ou le rayonnement électromagnétique.

Parmi les sondes les plus courantes, le neutron dit thermique est une des plus intéressantes et riches de possibilités. En fait, le neutron est une particule neutre et qui n'interagit donc pas avec les champs électriques, dont celui dû aux électrons. De ce fait, la pénétration dans la matière est très facile même avec des énergies cinétiques très faibles, souvent inférieures à celle de l'agitation thermique des systèmes étudiés. Les informations sur la structure et la dynamique des échantillons étudiés par diffusion de neutrons proviennent donc exclusivement des forces nucléaires entre les neutrons et les noyaux des atomes.

Une autre interaction importante est celle du neutron avec l'induction magnétique du milieu traversé, dont celle qui résulte d'électrons dépareillés dans les matériaux magnétiques. Cette introduction n'abordera pas l'interaction magnétique, malgré son énorme intérêt.

Pour saisir globalement la nature des interactions neutrons-matière, il est utile de rappeler quelquesunes des propriétés du neutron :

$$
\begin{aligned}
& \text { Masse }=1.675 \times 10^{-24} \mathrm{~g} \\
& \text { Charge }=0 \\
& \text { Durée de vie }: 886.8 \mathrm{~s}(14.8 \text { minutes }) \\
& \text { Spin }=\frac{1}{2} \\
& \text { Moment dipolaire : }-1.913 \mu_{B}
\end{aligned}
$$

Les expériences de diffusion de neutrons utilisent exclusivement des neutrons "thermiques", ce qui veut dire que leur vitesse a été préalablement diminuée ("modérée") par interaction inélastique avec un milieu "thermalisé" à des températures adéquates : $20 \mathrm{~K}$ (thermalisation à l'hydrogène liquide, neutrons "froids"), $300 \mathrm{~K}$ (neutrons "thermiques"), $1200^{\circ} \mathrm{C}$ (neutrons "chauds"). Dans ces domaines de basse énergie, il n'y a presque jamais lieu de tenir compte de termes de résonance ou des niveaux d'énergie des noyaux dans l'interaction neutron -noyau.

En tenant compte de la masse du neutron et des valeurs de la température, on peut facilement déduire les vitesses, $v$, des neutrons. La relation de de Broglie qui relie le moment d'une particule à celui d'une onde plane, donc à une longueur d'onde $(m v=h k / 2 \pi=h \lambda)$, permet d'écrire les relations suivantes, où E est l'énergie, $k_{B}$ la constante de Boltzmann, $T$ la température, $m$ la masse du neutron, $v$ sa vitesse et $\lambda$ la longueur d'onde.

$$
\begin{gathered}
E=\left(k_{B} T\right)=\frac{1}{2} m v^{2}=\left(\frac{h^{2}}{2 m}\right) \frac{1}{\lambda^{2}} \\
\lambda=6.283 \frac{1}{k}=3.956 \frac{1}{v}=9.045 \frac{1}{\sqrt{E}} \\
E=5.227 v^{2}=81.81 \frac{1}{\lambda^{2}}=2.072 k^{2}
\end{gathered}
$$

La relation énergie-température figure entre parenthèse parce que, physiquement elle devrait être égale à $3 k_{B} T / 2$, mais la "tradition" la fait écrire de cette manière. Cela est sans conséquences, puisque de 
toute manière les neutrons disponibles ont un spectre très large plus ou moins maxwellien et, en pratique, le paramètre intéressant est la longueur d'onde, souvent imposée par monochromatisation du faisceau incident, où déduite de la vitesse.

Il est important de constater les domaines numériques (voir le Tableau ci-dessous) des paramètres qui résultent des équations ci-dessus, pour les neutrons thermiques. La "fenêtre" de longueur d'onde s'étend de 0.5 à $20 \AA$ A , des valeurs comparables aux distances inter-atomiques ; la fenêtre d'énergie est, par construction, comparable aux énergies cinétiques. Ces deux caractéristiques rendent la diffusion de neutrons une technique de choix pour l'étude de la structure de la matière à l'échelle atomique, ainsi que pour l'étude des mouvements atomiques et moléculaires (diffusions, rotations, vibrations, phonons, etc.).

\begin{tabular}{|c|c|c|c|c|}
\hline $\begin{array}{c}\lambda \\
(\AA)\end{array}$ & $\begin{array}{c}v \\
(\mathrm{~km} / \mathrm{s})\end{array}$ & $\begin{array}{c}E \\
(\mathrm{meV})\end{array}$ & $\begin{array}{c}E \\
(\mathrm{~K})\end{array}$ & $\begin{array}{c}1 \AA \\
(\mathrm{ps})\end{array}$ \\
\hline 1 & 3.96 & 81.81 & 949 & 0.025 \\
\hline 4 & 0.99 & 5.11 & 59.3 & 0.101 \\
\hline 10 & 0.40 & 0.818 & 9.49 & 0.252 \\
\hline
\end{tabular}

Rappelons que, dans le cas de la diffusion de la lumière visible, la longueur d'onde est de l'ordre du $\mu m$ et que les rayons $X$ ont des énergies très supérieures à $k_{B} T$. Peut-être plus important est le fait que l'énergie varie avec le carré de la longueur d'onde offrant une plage d'étude en énergie très large. Dans le cas des ondes électromagnétiques, l'énergie dépend linéairement de la longueur d'onde.

Malgré donc de solides atouts, la diffusion de neutrons est le plus souvent une technique "complémentaire". Ceci résulte de la faible intensité des sources, des processus de thermalisation et de monochromation, des difficultés de focalisation des faisceaux. Les ordres de grandeur des flux moyens disponibles dans une installation moderne sont les suivants :

Flux au niveau du réflecteur du réacteur : $10^{15} \mathrm{n} \mathrm{cm}^{2} \mathrm{~s}^{-1}$

Flux polychromatique après thermalisation : $10^{9} \mathrm{n} \mathrm{cm}^{2} \mathrm{~s}^{-1}$

Flux monochromatique au niveau d'un échantillon : $10^{5}$ à $10^{7} \mathrm{n} \mathrm{cm}^{2} \mathrm{~s}^{-1}$

Ces valeurs sont plusieurs ordres de grandeur inférieures à celles disponibles dans les installations courantes de diffusion de la lumière ou de rayons $X$, sans parler des grandes installations actuelles de rayonnement synchrotron. La conséquence logique est que la diffusion neutronique est réservée à des cas qui justifient absolument son utilisation, ce qui, pourtant, ne devrait pas limiter les demandes de temps de faisceau et la bonne apprentissage de la technique!

Si l'on part du flux disponible au niveau d'un échantillon, évoqué ci-dessus, on se rend compte que, pour un échantillon typique contenant environ $10^{22}$ atomes, il faudrait attendre des millions d'années avant que chaque site ait interagit avec un neutron. En réalité, la distance entre les neutrons dans le faisceau incident est très grande (de l'ordre $\mathrm{du} \mathrm{cm}$ ) et chacun peut être pris comme une particule classique dont on connaît et la position et le moment. Mais, dans l'échantillon, la distance typique entre atomes étant, comme nous l'avons vu, comparable à la longueur d'onde, on se trouve dans la situation où on connaît le moment du neutron (module de la vitesse imposée par la monochromatisation, direction imposée par la collimation) mais on ne connaît pas la position. Idéalement, chaque neutron est une onde plane d'extension infinie qui interagit avec tous les noyaux de l'échantillon. En pratique, l'extension est finie et imposée par la collimation (cohérence transversale) ce qui définit une "aire de cohérence" à l'intérieur de laquelle les déphasages dus aux interactions avec chaque atome peuvent être interprétés en termes de distances inter-atomiques. Pour les appareillages courants, cette aire de cohérence est de l'ordre des $\mu \mathrm{m}^{2}$. Notons que, néanmoins, le neutron est presque toujours détecté en tant que particule. L'interférence constructive "régénère" le neutron avec une loi de probabilité qui dépend des interactions et de la structure de l'échantillon. Une expérience visant la détermination de la structure doit se faire 
de manière à que l'aire de cohérence soit plus grande que les distances en jeu pour la définition de la structure à une échelle donnée.

Remarque : Ceci n'a strictement rien à voir avec la "résolution" expérimentale. Les faibles intensités des flux disponibles amènent à utiliser des neutrons dont la longueur d'onde est à l'intérieur d'une bande dont la largeur atteint parfois $10 \%$ de la longueur d'onde nominale, ce qui amène à une certaine résolution structurale. Mais, dans l'interaction neutron-matière, chaque neutron doit être pris individuellement.

La théorie de la diffusion des neutrons par les noyaux atomiques est assez simple du fait que ces derniers peuvent être considérés comme ponctuels $\left(10^{-17} \mathrm{~cm}\right)$. Le potentiel d'interaction est donc sphérique. La valeur typique de la section efficace de diffusion est de $10^{-24} \mathrm{~cm}^{2}=1$ barn.

Les détails de la théorie se trouvent dans plusieurs livres et articles de synthèse. On ne rappellera ici que le processus de l'interaction, idéalisé pour un atome isolé. L'onde plane incidente interagit avec le noyau et génère une onde sphérique d'amplitude $b$ (la longueur de diffusion cohérente) :

La section efficace cohérente est donnée par $\sigma_{c o h}=4 \pi b^{2}$.

Notons que cette représentation est schématique dans la mesure où, en pratique, la valeur de $b$ correspond à un atome "lié", c'est-à-dire, parmi les autres atomes de la matière.

Ce sont les interférences entre les ondes sphériques issues de plusieurs atomes qui, d'une manière bien connue, vont générer des interférences constructives dans certaines directions de l'espace.

Dans le schéma ci-dessus $b$ est affecté d'un indice \pm . Comme nous avons vu au début, le neutron a un spin 1/2. Chaque noyau a un spin $I$. Dans l'interaction de l'onde plane avec chaque noyaux le couplage peut se faire avec les spins parallèle (spin résultant $I+1 / 2$ ) ou anti-parallèle (spin résultant $I-1 / 2$ ). Dans chaque cas, l'interaction sera différente et il $y$ a lieu, pour chaque noyau (chaque isotope) de définir deux valeurs de $b: b_{+}$(spins parallèles) et $b_{-}$(spins anti-parallèles), les seules exceptions étant les noyaux de spin nul. Certaines expériences utilisent des faisceaux "polarisés" où tous les spins des neutrons incidents ont la même direction suite à l'interaction avec un milieu polarisant. Dans d'autres cas, les spins nucléaires peuvent eux aussi être polarisés. La considération de ces cas dépasse le caractère élémentaire de cette introduction. Notons que, dans le cas le plus fréquent, ni les spins des neutrons incidents ni ceux de l'échantillon ne sont orientés. C'est donc "le même" neutron (onde plane, comme nous l'avons vu) que, de manière aléatoire se couple avec chaque noyau, la statistique obéissant, bien sûr, aux règles de la mécanique quantique.

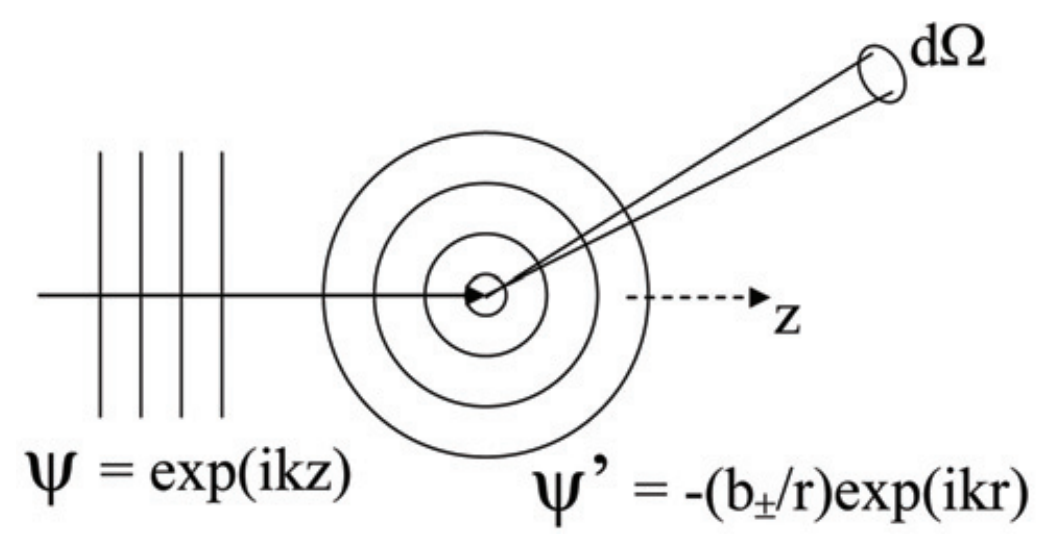

En pratique, on définit pour chaque isotope une valeur moyenne des longueurs de diffusion cohérent, moyenne pondérée de $b_{+}$et de $b_{-}$. C'est la valeur de la "longueur de diffusion cohérente" que l'on trouve sur les Tables. Pour les éléments naturels, on calcule aussi un $b$ moyen pondéré par la concentration isotopique. Les moyennes des écarts à ces valeurs définissent une "section efficace incohérente". 
La section efficace de diffusion d'un noyau est donc la somme d'un terme cohérent et d'un terme incohérent.

A titre d'exemple, le tableau suivant indique les valeurs des différentes valeurs évoquées pour l'hydrogène et pour le deutérium.

Spin of the nucleus-neutron system

Number of states

Scattering length

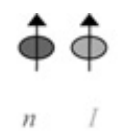

$\mathrm{I}+1 / 2$

$f .=\frac{I+1}{2 I+1}$

$\mathrm{b}+$

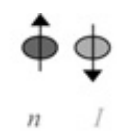

$\mid \mathrm{I}-1 / 2$ |

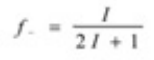

b.

$$
\begin{aligned}
& \bar{b}=f_{+} b_{+}+f_{-} b_{-} \\
& \overline{b^{2}}=f_{+} b_{+}^{2}+f_{-} b_{-}^{2} \\
& \sigma_{c o h}=4 \pi(\bar{b})^{2} \\
& \left.\sigma_{i n c}=4 \pi \overline{b^{2}}-(\bar{b})^{2}\right] \\
& \sigma_{s}=\sigma_{c o h}+\sigma_{i n c}
\end{aligned}
$$

\begin{tabular}{|c|c|c}
\hline & H & D \\
\hline $\mathrm{I}$ & $1 / 2$ & 1 \\
\hline $\mathrm{I}+1 / 2$ & 1 & $3 / 2$ \\
\hline $\mathrm{I}-1 / 2$ & 0 & $1 / 2$ \\
\hline $\mathrm{b}+\left(10^{-12} \mathrm{~cm}\right)$ & 1.085 & 0.953 \\
\hline $\mathrm{b} \cdot\left(10^{-12} \mathrm{~cm}\right)$ & -4.750 & 0.098 \\
\hline $\mathrm{f}_{+}$ & $3 / 4$ & $2 / 3$ \\
\hline $\mathrm{f}$. & $1 / 4$ & $1 / 3$ \\
\hline$b\left(10^{-12} \mathrm{~cm}\right)$ & -0.374 & 0.668 \\
\hline$b^{2}($ barn $)$ & 6.523 & 0.609 \\
\hline$\sigma_{\text {cob }}$ (barn) & 1.758 & 5.607 \\
\hline$\sigma_{\text {imc }}$ (barn) & 79.91 & 2.04 \\
\hline$\sigma_{s}$ (barn) & 81.67 & 7.65 \\
\hline
\end{tabular}

$\left(1\right.$ barn $\left.=10^{-24} \mathrm{~cm}^{-2}\right)$

Le cas de l'hydrogène est unique en ce qui concerne la section efficace incohérente. Le plus souvent les études structurales de matériaux hydrogénés sont difficiles, entachées d'erreurs inhérentes, voire impossibles. Mais, elle ouvre la possibilité de l'étude très précise de dynamiques individuelles de matériaux hydrogénés, puisque toute contribution cohérente $y$ est négligeable.

Notons enfin que la substitution isotopique, se traduisant éventuellement par des diffusions cohérentes diverses, ouvre des possibilités de "marquage" d'atomes, de molécules ou de fragments d'agrégats d'une grande richesse potentielle.

Les études structurales s'appuient sur l'hypothèse dite "approximation statique". Elle admet que l'onde qui parcourt l'échantillon interagit avec eux dans le volume de cohérence pendant des temps courts par rapport aux temps caractéristiques de la matière. Au contraire de ce qui arrive dans le cas des ondes électromagnétiques, cette hypothèse n'est pas toujours bien vérifiée. En effet, pour des neutrons de $8 \AA$, la vitesse est égale à $500 \mathrm{~ms}^{-1}$, ce qui correspond à $5 \AA$ A $/$ ps (voir le tableau ci-dessus). Or, beaucoup de mouvements moléculaires (diffusion, rotation) ont lieu sur des temps de l'ordre de la ps. Il serait intéressant d'aborder dans certains cas les conséquences qui en résultent en cas de violation.

La théorie détaillée de la diffusion élastique se trouve dans beaucoup de travaux de référence. Notons qu'elle a ses spécificités, hypothèses et terminologie dans chaque grand domaine d'étude de la matière : 
cristallographie de monocristaux et de poudres, structures de liquides et de verres, macromolécules et colloïdes en suspension, polymères, matériaux sous champ magnétique ou sous cisaillement, etc.

Le tableau suivant donne la formule de base de l'intensité diffusée $I(Q, t)$, ainsi que l'approximation statique, point de départ de toute étude structurale puisqu'elle fournit $S^{c o h}(Q)$, soit le facteur de structure :

Approximation statique :

$$
\begin{aligned}
I(\vec{Q}, t)= & \sum_{i, j} b_{i} b_{j}\left\langle\exp \left[i \vec{Q} \cdot \vec{r}_{i}(0)\right] \cdot \exp \left[-i \vec{Q} \cdot r_{j}(t)\right]\right\rangle \\
& +\sum_{i}\left(b_{i}^{i n c}\right)^{2}\left\langle\exp \left[i \vec{Q} \cdot \vec{r}_{i}(0)\right] \cdot \exp \left[-i \vec{Q} \cdot \vec{r}_{i}(t)\right]\right\rangle
\end{aligned}
$$

$$
S^{c o h}(\vec{Q})=\sum_{i, j} b_{i} b_{j} \exp \left[i \vec{Q} \cdot\left(\vec{r}_{i}-\vec{r}_{j}\right)\right]+\sum_{i}\left(b_{i}^{i n c}\right)^{2}
$$

Dans ces expressions $r_{i}(t)$ dénote la position du noyau $i$ au temps $t$. L'approximation statique consiste à négliger la dépendance temporelle de $r_{i}$, le temps de l'interaction.

Dans ces expressions $Q$, comme d'habitude est l'échange de moment. Ci-dessous nous rappelons les définitions géométriques en mettant en relief les cas élastique (sans échange d'énergie) et inélastique.

Notons dans les deux premiers schémas que l'on peut représenter la diffusion neutronique soit dans l'espace réel soit dans l'espace réciproque. Dans le premier cas, le neutron incident peut être considéré (comme nos l'avons vu) comme une particule classique de vitesse $v$ (ou comme un groupe d'ondes). De même, en ce qui concerne les neutrons émergeants détectés par un détecteur à une distance grande par rapport à la taille de l'échantillon. Vu le nombre de neutrons impliqués, le transfert de moment macroscopique est négligeable. Dans une représentation dans l'espace réciproque, ce sont les moments

$$
|\mathrm{k}|=2 \pi / \lambda \quad E=\hbar^{2} k^{2} / 2 m
$$

Espace réel:

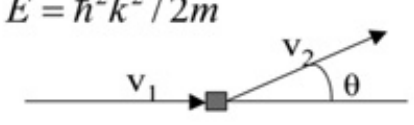

Echantillon

\section{Espace réciproque:}
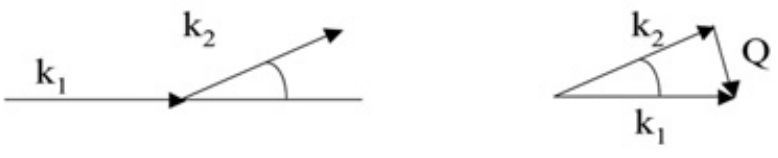

1) Diffusion élastique

$$
\begin{aligned}
\left|k_{1}\right| & =\left|k_{2}\right| \\
E_{1} & =E_{2}
\end{aligned}
$$

$Q=(4 \pi / \lambda) \sin (\theta / 2)$

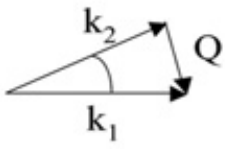

2) Diffusion inélastique

$$
\begin{gathered}
\left|\mathrm{k}_{1}\right| \neq\left|\mathrm{k}_{2}\right| \\
\hbar \omega \equiv E_{1}-E_{2} \neq 0
\end{gathered}
$$$$
Q=\sqrt{k_{1}^{2}+k_{2}^{2}-2 k_{1} k_{2} \cos \theta}
$$

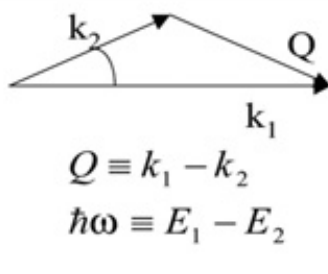


que figurent et l'échantillon est alors réduit à un point, vu ses dimensions macroscopiques. Beaucoup de représentations mélangent parfois des éléments des espaces réel et réciproque, rendant la théorie inutilement confuse.

Pour donner des exemples illustrant les possibilités de la diffusion neutronique dans un cas académique, nous montrons ci-dessous le cas d'un système contenant deux types de particules (deux valeurs de $b$ ou de densité de $b$ différentes). On $y$ décèle, a priori, des fluctuations en nombre et des fluctuations des concentrations locales des deux espèces. Ainsi, dans le premier cas, les "fluctuations de densité" font apparaître à chaque instant des distributions spatiales des espèces non homogènes. Ceci est d'ailleurs à l'origine d'une compressibilité isotherme. Par ailleurs, les potentiels entre les deux types de particules étant différents, on aura aussi des régions de ségrégation, soit des "fluctuations de concentration" qui expliquent la compressibilité osmotique. Il est clair qu'un marquage isotopique convenable peut éliminer la différence de section efficace de diffusion cohérente entre les deux types de particules (via des mélanges isotopiques convenables). Alors, l'intensité diffusée ne mesurera que les fluctuations de densité.
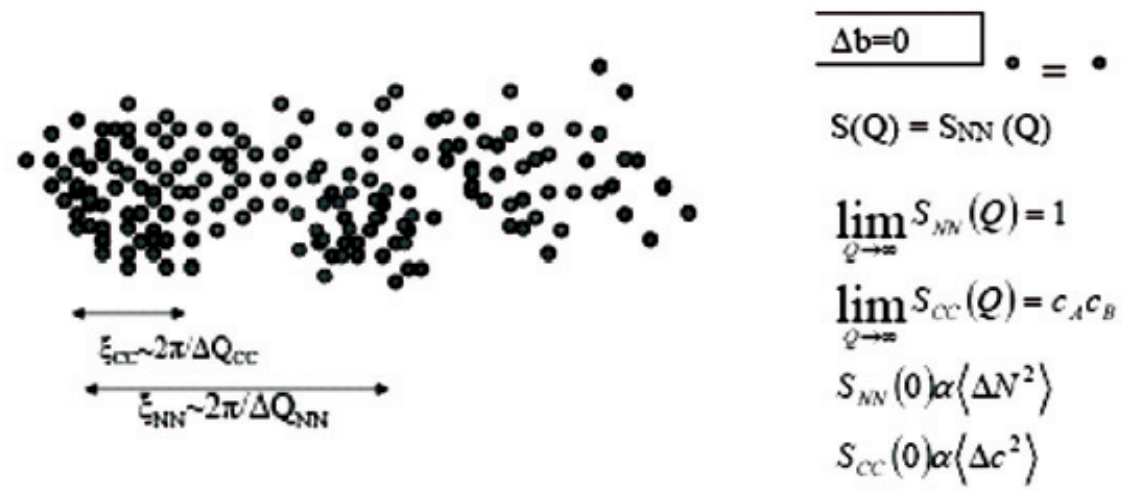

Bien sûr, ce type d'approche est mis à profit dans des situations très diverses, telles que la détermination de facteurs de structure partiels, de parties de macromolécules, de conformation de polymères, etc.

En ce qui concerne la dynamique, les neutrons pâtissent des conséquences indirectes de la faible énergie des neutrons du faisceau incident. La dépendance intrinsèque $\omega(Q)$ qui relie le transfert de moment $Q$ et le transfert d'énergie $\omega$ pour une longueur d'onde donnée, implique que le côté "perte d'énergie" (des neutrons) est limité à l'énergie (faible, naturellement) des neutrons incidents. Or, ce côté est celui de la diffusion Stokes, où l'intensité des modes inélastiques est plus grande. D'ailleurs, à très basse température, le côté anti-Stokes peut être pratiquement inexistant. Aussi, dans certains calculs, par exemple, de détermination de la densité d'états vibrationnels, la prise en compte de la courbe de dispersion des neutrons n'est pas toujours aisée. Malgré cette limitation, les études de dynamique font souvent appel aux neutrons à cause de ses avantages intrinsèques : séparation des dynamiques individuelles et collectives, obtention des courbes de dispersion des excitations collectives, études d'excitations magnétiques, théorie simple, c'est à dire, ne faisant pas appel à des fonctions de couplage mal connues.

Le diagramme vectoriel suivant montre le rapport entre les échanges de moment et d'énergie dans une expérience de diffusion inélastique ainsi que la courbe de dispersion qui en résulte.

Le tableau suivant décrit les situations, dites de gain et de perte d'énergie des neutrons et, schématiquement une composante inélastique.

Un cas particulier de la diffusion neutronique est la réflexion (spéculaire ou pas). Il sera développé par d'autres intervenants.

La réflexion totale résulte de l'interférence entre des ondes diffusées aux petits angles et l'onde incidente. C'est un des rares cas de diffusion de neutrons thermiques où l'interaction globale n'est pas faible. Le cas intéressant est celui d'une surface pour laquelle la valeur moyenne de $b$ est positive. 

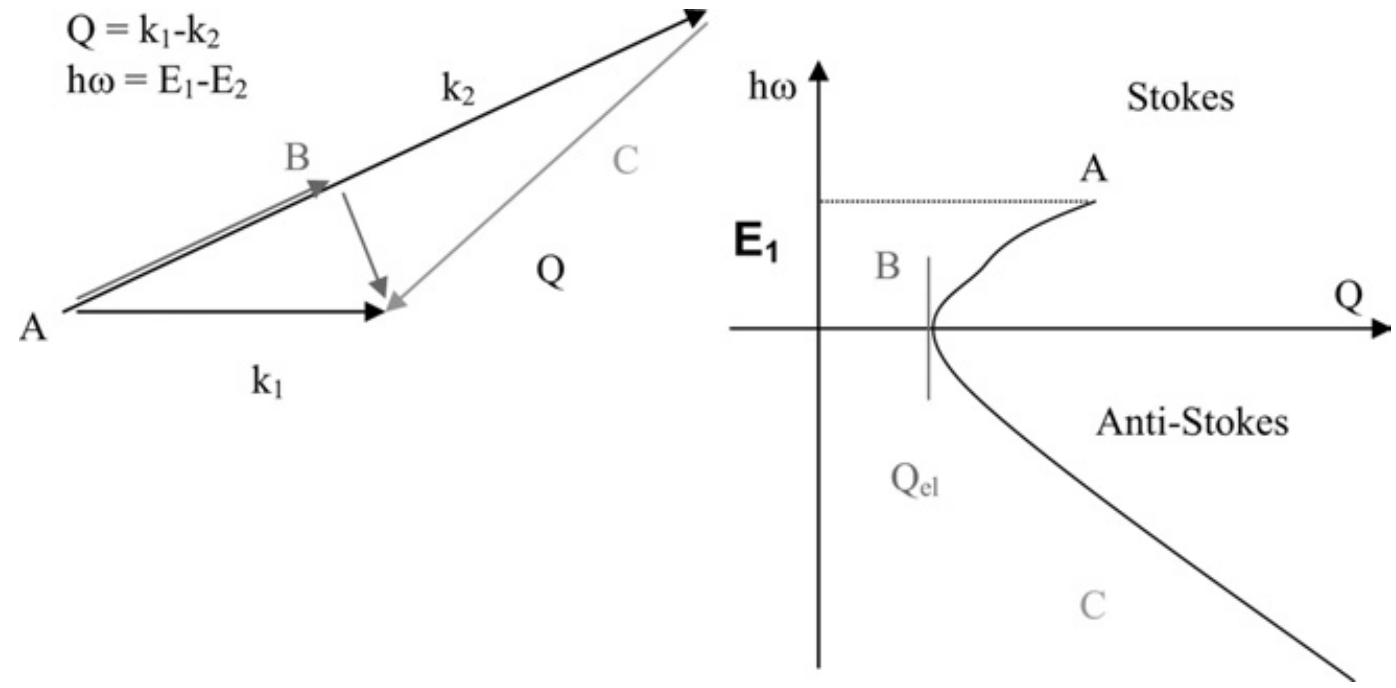

$\hbar \omega=E_{1}-E_{2}$

\begin{tabular}{|c|c|c|c|}
\hline$\hbar \omega$ & $\begin{array}{l}\text { Energy of } \\
\text { the } \\
\text { neutron }\end{array}$ & $\begin{array}{l}\text { Energy of } \\
\text { the } \\
\text { system }\end{array}$ & \\
\hline$>0$ & loss & gain & Stokes \\
\hline$<0$ & gain & loss & $\begin{array}{l}\text { Anti- } \\
\text { Stokes }\end{array}$ \\
\hline
\end{tabular}

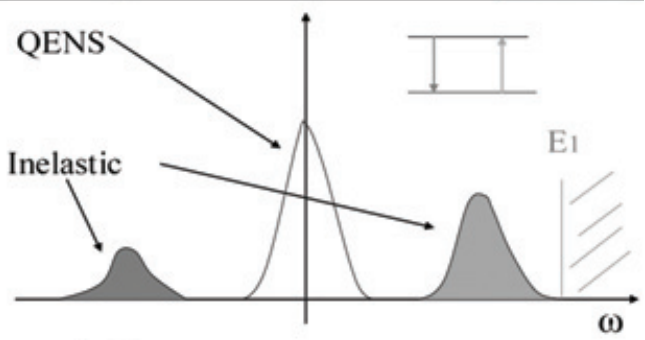

Anti-

Stokes

Stokes

Pour une lamelle de faible épaisseur $t$, l'onde diffusée (S) et l'onde incidente (I) sont en quadrature et la résultante (R) a un déphasage avec I égal à $\Phi=N \lambda \rho t$. Le rapport entre $\Phi$ et l'indice $n$ est donné par :

$$
\Phi=\left(\frac{2 \pi}{\lambda}\right)(I-n) t
$$

On en déduit :

$$
n=1-N \lambda^{2} \rho
$$

L'angle est donné par $\cos \theta_{c}=n$, soit

$$
\theta_{c}=\lambda \sqrt{\frac{N \rho}{\pi}}
$$


Le tableau suivant indique les diverses fonctions que l'on peut définir dans l'espace réel $(r)$, réciproque $(Q)$, en fonction du temps $(t)$ ou de l'énergie $(\omega)$. Les appareils mesurent $S(Q), S(Q, \omega)$ ou $I(Q, t)$ avec une richesse très grande de caractéristiques et de possibilités. On passe d'une fonction à l'autre par des transformations linéaires, les transformées de Fourier dans le temps et dans l'espace.

\section{Van Hove function}



Enfin les tableaux suivants donnent un aperçu de l'extrêmement large domaine d'applications de la diffusion neutronique. 


\section{STRUCTURE S(Q)}

\begin{tabular}{|c|c|}
\hline Structure de monocristaux & 4 cercles \\
\hline $\begin{array}{l}\text { Structure de poudres } \\
\text { (y compris, structures } \\
\text { magnétiques) }\end{array}$ & $\begin{array}{l}2 \text { axes haute et moyenne } \\
\text { résolution }\end{array}$ \\
\hline Défauts dans les solides & $\begin{array}{l}\text { Diffusion diffuse ("Diffuse } \\
\text { scattering") }\end{array}$ \\
\hline $\begin{array}{l}\text { Structure de liquides et de } \\
\text { verres? } g(r)\end{array}$ & 2 axes (neutrons "chauds") \\
\hline $\begin{array}{l}\text { Structure de macromolécules } \\
\text { (dont protéïnes) }\end{array}$ & Petits angles haute résolution \\
\hline $\begin{array}{l}\text { Structures nanoscopiques } \\
\text { (1 à } 100 \mathrm{~nm} \text { ) } \\
\text { (précipités, grains } \\
\text { magnétiques, polymères, } \\
\text { colloïdes, membranes,...) }\end{array}$ & Petits angles (SANS) \\
\hline $\begin{array}{l}\text { Structures mésoscopiques } \\
(10 \text { à } 1000 \mathrm{~nm})\end{array}$ & Très petits angles (USANS) \\
\hline $\begin{array}{l}\text { Profils de densité et } \\
\text { d'aimantation }\end{array}$ & Réflectomètre (spéculaire) \\
\hline Structures à 2 dimensions & Réflectomètre (non spéculaire) \\
\hline Déformations /Contraintes & Contraintes ("Stress") \\
\hline \begin{tabular}{|l|} 
Textures \\
\end{tabular} & 4 cercles \\
\hline
\end{tabular}

\section{DYNAMIQUE $\mathrm{S}(\mathrm{Q}, \omega) \quad \mathrm{I}(\mathrm{Q}, \mathrm{t})$}

\section{A) Collective}

\begin{tabular}{|l|l|}
\hline $\begin{array}{l}\text { Phonons, modes acoustiques } \\
\text { dans les liquides }\end{array}$ & 3 axes \\
\hline Magnons & 3 axes (neutrons polarisés) \\
\hline
\end{tabular}

B) Individuelle ("self")

\begin{tabular}{|l|l|}
\hline $\begin{array}{l}\text { Dynamique moléculaire } \\
\text { (diffusion, rotation) } \\
\text { (10-13 à 10-10 s) }\end{array}$ & Temps de vol \\
\hline $\begin{array}{l}\text { Dynamique moléculaire } \\
\text { (10-11 à 10-8 s) }\end{array}$ & $\begin{array}{l}\text { Retrodiffusion ("back- } \\
\text { scattering") }\end{array}$ \\
\hline $\begin{array}{l}\text { Dynamique moléculaire et } \\
\text { mésoscopique } \\
\text { (cohérente) }\end{array}$ & Echo de spin \\
\hline Densité d'états de vibration & Temps de vol \\
\hline
\end{tabular}

